\title{
Efectos de la contratación de exempleados de multinacionales en la capacidad de absorción en empresas locales
}

\author{
Effects of hiring former employees of multinationals in the absorptive \\ capacity of local firms \\ Juan Carlos Leiva ${ }^{\mathrm{a}, *}$, Juan Antonio Rodríguez Álvarez ${ }^{\mathrm{a}, \mathrm{b}}$ \\ y Ricardo Monge González ${ }^{\mathrm{a}}$ \\ a Tecnológico de Costa Rica, Costa Rica \\ b Universidad de Costa Rica, Costa Rica
}

Recibido el 29 de septiembre de 2015; aceptado el 17 de junio de 2016

Disponible en Internet el 1 de marzo de 2017

\section{Resumen}

La atracción de inversión extranjera directa busca, entre otros objetivos, aumentar la productividad de las empresas locales vía derrames de conocimiento. Sin embargo, la evidencia empírica al respecto es contradictoria. Un factor influyente es la capacidad de absorción de las empresas locales. Este artículo analiza el efecto de la presencia de exempleados de empresas multinacionales, como empleados de empresas locales, en la capacidad de absorción de dichas empresas. El estudio se realizó en Costa Rica, país reconocido por su exitosa estrategia en la materia. Los datos provienen de una encuesta a 1,167 empresas efectuada por el Observatorio Costarricense de las Pymes en 2011. Se halló que la contratación de exempleados de empresas multinacionales por parte de las empresas locales tiene un efecto positivo sobre el índice de capacidad de absorción de conocimiento en las empresas de todos los sectores productivos. Específicamente, esta

\footnotetext{
* Autor para correspondencia.

Correo electrónico: jleiva@tec.ac.cr (J.C. Leiva).

La revisión por pares es responsabilidad de la Universidad Nacional Autónoma de México.
} 
contratación de exempleados incrementa en nueve puntos porcentuales el índice de capacidad de absorción, con diferencias por sectores y tamaños de empresas.

(C) 2017 Universidad Nacional Autónoma de México, Facultad de Contaduría y Administración. Este es un artículo Open Access bajo la licencia CC BY-NC-ND (http://creativecommons.org/licenses/by-nc-nd/4.0/).

Palabras clave: Capacidad de absorción; Empresas multinacionales; Pymes; Derrames de conocimiento; Costa Rica Códigos JEL: M21; O19; F23

\section{Abstract}

Attracting Foreign Direct Investment seeks, among other goals, increase productivity of local firms through knowledge spillovers. Nevertheless the empirical evidence is contradictory. One influential factor is the absorptive capacity of local firms. This article analyzes the effect of the presence of ex-employees of multinational companies, as employees of local firms in the absorption capacity of these firms. The study was conducted in Costa Rica, a country recognized for its successful strategy in this area. The data come from a survey of 1,167 companies conducted by the Costa Rican Observatory of Small and Mediums Enterprises in 2011. It was found that hiring former employees of multinational companies by local firms has a positive effect on the rate of absorption capacity. In short, this engagement of former employees of multinationals increases by nine percentage points the rate of absorption capacity, with differences for sectors and firms sizes.

(C) 2017 Universidad Nacional Autónoma de México, Facultad de Contaduría y Administración. This is an open access article under the CC BY-NC-ND license (http://creativecommons.org/licenses/by-nc-nd/4.0/).

Keywords: Absorption capacity; Multinationals companies; SME; Knowledge spillovers; Costa Rica JEL classification: M21; O19; F23

\section{Introducción}

La atracción de inversión extranjera directa mediante el establecimiento de empresas multinacionales (EMN) es una estrategia por la cual han apostado muchos países, esperando con ello obtener, entre otros beneficios, derrames de conocimiento desde dichas EMN (quienes se ubican más cerca del estado del arte en su materia) hacia las empresas locales y así aumentar la productividad de estas últimas (Javorcik, 2004; Görg y Strobl, 2005; Smeets, 2008). Existen tres canales por medio de los cuales pueden ocurrir derrames de conocimiento desde las multinacionales hacia las empresas locales: a) efectos demostración, los cuales incluyen la imitación, o ingeniería inversa de los productos o prácticas de las EMN por parte de las empresas locales; b) movilidad laboral, que permite a los empleados que han sido capacitados por EMN aplicar sus conocimientos en las empresas locales, cuando ellos dejan de trabajar en las primeras, y c) encadenamientos verticales hacia delante y hacia atrás entre EMN y sus proveedores locales (Saggi, 2002). En este artículo nuestro interés se centra en uno de ellos: la movilidad laboral. Esto por cuanto ella permite, a los empleados que han sido capacitados por EMN, aplicar sus conocimientos en las empresas locales donde pasan a trabajar, cuando dejan de hacerlo en las primeras.

No obstante, hasta el momento la evidencia empírica sobre los resultados de esa estrategia de atracción de inversión extranjera directa en el conglomerado empresarial del país anfitrión sigue siendo ambigua (Zhang, Li, Li y Zhou, 2010). Ante ello, el estudio de los factores determinantes para que se produzcan dichos derrames de conocimiento es relevante tanto para académicos y practicantes como para diseñadores de política pública. 
Uno de los factores que ha surgido para buscar comprender el fenómeno descrito es la capacidad de absorción (CA) de las empresas locales. Dicha CA se define como aquel conjunto de rutinas y procesos por medio de los cuales las organizaciones adquieren, asimilan, transforman y explotan conocimiento de su entorno para producir una capacidad organizacional dinámica (Zahra y George, 2002). Por ende, la capacidad que tengan las empresas locales para absorber el conocimiento derramado desde las EMN es nuestro foco de interés.

Es importante mencionar que dicha CA se ha convertido en un tema de estudio desde diversas facetas. Por ejemplo, ha sido ligada con diferencias en el desempeño de empresas según diversos entornos (Liao, Welsch y Stoica 2003; Wales, Parida y Patel, 2013); con la creación de empresas a partir del conocimiento generado por otras empresas (Qian y Acs, 2013), y con el desempeño de empresas locales en ámbitos donde coexisten con firmas multinacionales (Zhang et al., 2010; Girma y Görg, 2002). A su vez, han existido diversos enfoques para intentar comprender los factores que influyen en la CA, sus componentes como tal y sus interacciones con otros elementos del entorno económico (Camisón y Forés, 2010; Qian y Acs, 2013).

En este ámbito es donde puede ubicarse el presente artículo. Nuestro objetivo es comprender los efectos que produce la contratación de exempleados de compañías multinacionales en la CA de las empresas locales. Esto resulta de interés desde diversas perspectivas. Si la CA es importante para el desempeño de las empresas como parece señalar la literatura científica (Wales et al., 2013), existen sobradas razones para buscar comprender «cómo se comporta» esa CA y qué factores influyen en su composición. En tal sentido es importante mejorar la comprensión del rol que juega un factor muy concreto, la presencia de exempleados de EMN, en la CA de las empresas locales del país anfitrión donde operan las EMN. Esto además permite valorar uno de los beneficios que se espera con la atracción de inversión extranjera directa en muchos países: el derrame de conocimientos hacia las empresas locales.

El estudio se aplicó en Costa Rica, un país que ha sido reconocido internacionalmente por su exitosa estrategia de atracción de inversión extranjera directa (Cordero y Paus, 2008; World Economic Forum, 2013), que se combina con una amplia base de empresas locales donde predominan las micros, pequeñas y medianas (mipymes) (Gómez, 2012) En este caso particular, Monge-González y González-Alvarado (2007) ilustran la contribución de las EMN de alta tecnología en el desarrollo de habilidades por parte de los trabajadores costarricenses, con base en las experiencias de Intel, Microsoft y Cisco. Además, Monge-González, Leiva y Rodríguez-Álvarez (2012) documentan la alta movilidad laboral desde las EMN hacia las empresas costarricenses durante el período 2001-2007, así como el papel de dichas EMN como fuente de adquisición de habilidades «blandas» para sus empleados.

En función del objetivo planteado, el documento se ha organizado en cinco secciones, incluida la introducción. En la segunda sección se presenta el marco referencial teórico, en la tercera sección se aborda el marco referencial metodológico, en la cuarta sección se discuten los resultados empíricos, y en la quinta y última sección se formulan las principales conclusiones del estudio.

\section{Marco teórico}

La gestión del conocimiento es un enfoque conceptual que cataloga al conocimiento como el recurso más importante, estratégicamente hablando, que posee una firma (Grant, 1996). En particular, sus estudiosos se han orientado a dilucidar cómo es creado, compartido y usado el conocimiento en las organizaciones (Nonaka, 1994), de forma tal que se convierta en ventaja competitiva sostenible de ellas (Ragab y Arisha, 2013; Teece, Pisano y Shuen, 1997). Es bajo 
este paradigma teórico que se ubica nuestro trabajo, intercalando aspectos de CA y desempeño de empresas locales, afectado por las EMN que operan en la economía anfitriona.

\section{Concepto y medición de la capacidad de absorción}

En su concepción original la CA se entiende como la habilidad de una empresa para reconocer el valor de información nueva, externa a la empresa, para asimilarla y aplicarla con fines comerciales (Cohen y Levinthal, 1990). Posteriores refinamientos han conducido a un concepto de CA en cuatro componentes: adquirir, asimilar, transformar y explotar el conocimiento con el fin de generar una capacidad organizacional dinámica (Zahra y George, 2002). En este sentido, dichos autores definen a la CA como un conjunto de rutinas y procesos. Además, los cuatro componentes se agrupan en dos grandes dimensiones: capacidad potencial (adquisición y asimilación) y capacidad realizada (trasformación y explotación).

En cuanto a la forma de medir o cuantificar la CA, en la literatura científica se notan varios abordajes. En algunos trabajos se ha medido la CA por medio de escalas construidas ad hoc, mediante revisiones teóricas y análisis estadísticos. Por ejemplo, se han construido escalas para las variables ligadas a las cuatro dimensiones teóricas de adquisición, asimilación, transformación y explotación (Jansen, van den Bosch y Volberda, 2005; Camisón y Forés, 2010; Jiménez, García y Molina, 2011). Incluso algunos trabajos proponen nuevas dimensiones o constructos y diseñan la respectiva escala para medirlo. Muestra de ello es Lichtenthaler (2009), quien elabora una escala para medir la CA desde tres dimensiones (explorar, transformar y explotar) así como seis constructos, dos para cada dimensión, respetivamente (reconocer y asimilar; mantener y reactivar; transmutar y aplicar). De igual forma Vega-Jurado, Gutiérrez-Gracia y Fernández-de-Lucio (2008) proponen una escala para cuantificar un conjunto de determinantes o antecedentes de la CA que agrupan en tres dimensiones: conocimiento organizacional, formalización y mecanismos de integración social.

Por otro lado, existen una serie de propuestas para medir la CA por medio de indicadores más sencillos, de carácter unidimensional (proxy), como pueden ser gastos en investigación y desarrollo (I+D) (Rothaermel y Alexandre, 2009); gastos en I+D y experiencia de los empleados (Grimpe y Sofka, 2009), así como gasto e intensidad en I+D (De Jong y Freel, 2010).

Otro enfoque ha sido medir la CA por medio de una combinación de variables buscando una especie de índice, por ejemplo, gasto interno en I+D, la presencia de I+D permanente, el entrenamiento del personal de I+D y el porcentaje de científicos e investigadores de la organización (Escribano, Fosfuri y Tribó, 2009). En la misma línea, Murovec y Prodan (2009) midieron la CA mediante un constructo bidimensional según la fuente de información, ya fuera científica y de mercado, que utiliza la empresa. Por su parte, Monge-González, Rodríguez-Álvarez y Leiva (2015) miden la CA para las micros, pequeñas y medianas empresas costarricenses por medio de un índice construido a partir de variables emanadas de una encuesta y agrupando tales variables según los grupos determinantes propuestos por Vega-Jurado et al. (2008). En la sección metodológica se profundizará un poco en dicho índice, que fue utilizado para el presente artículo.

\section{Capacidad de absorción y desempeño de las empresas}

La CA ha sido ligada con algunas diferencias en el desempeño de las empresas, tanto de forma directa como indirecta por intermedio de otras variables. Es decir, operando como una variable mediadora.

En primera instancia los principales exponentes de la CA han esbozado teóricamente una relación lineal entre ella y el desempeño de las empresas (Cohen y Levinthal, 1990; Zahra y George, 
2002). En los trabajos empíricos más recientes se asocia la CA con el desempeño empresarial, pero desde una perspectiva que ve a la CA como una variable mediadora. Concretamente entre las actividades de internacionalización y el rendimiento financiero (Zahra y Hayton, 2008); el aprendizaje relacional (relationship learning) y el desempeño innovador (Chen, Lin y Chang, 2009); la mezcla de fuentes de tecnología (tecnología nueva vs conocida) y el rendimiento financiero (Rothaermel y Alexandre, 2009), así como las alianzas estratégicas y el rendimiento innovador y financiero (George, Zahra, Wheatley y Khan, 2001).

En un interesante trabajo, Wales et al. (2013) hallan una relación curvilínea (en forma de U invertida) entre la CA y el desempeño financiero, alcanzándose el máximo nivel de rendimiento financiero cuando la CA tiene un nivel intermedio. Además, su efecto fue moderado por otra variable denominada orientación emprendedora. Para llegar a estos resultados, dicho autores realizaron una investigación entre 285 pequeñas y medianas empresas (Pymes) suecas de base tecnológica. En su razonamiento los autores sostienen que esa relación curvilínea puede explicarse por el aumento incremental de los costos asociado con el aumento de la CA en las empresas. En otras palabras, a partir de cierto momento el costo marginal es mayor que el beneficio marginal de incrementar la CA.

\section{Factores que inciden sobre la capacidad de absorción}

Visto desde otra perspectiva, la CA como tal es influenciada por diversas variables del ámbito de las empresas. Es decir, ya la mira no se pone en la relación de CA con el desempeño, sino en qué factores influyen en la CA de las organizaciones.

Dentro de este enfoque, Vega-Jurado et al. (2008) proponen que la CA es determinada por tres tipos de variables: conocimiento organizacional, formalización y mecanismos de integración social. En su propuesta dichos autores esgrimen que estos tres factores se conjugan junto con el conocimiento externo relevante y se produce como resultado un nivel específico de CA. Por otro lado, Flor, Oltra y García (2011) encuentran que la CA varía según el tipo de estrategia que use la empresa. Para ello se basan en la tipología de Miles y Snow (1978), encontrando diferencias entre la CA (diferenciada entre potencial y realizada) y los tipos de estrategia de las empresa en estudio (exploradoras, defensoras, analizadoras y reactivas). Por su lado Jansen et al. (2005) ligan la CA con las capacidades organizacionales de coordinación, sistemas y socialización, mientras que Liao, Fei y Chen (2007) con la interacción que tienen los empleados con su propio conocimiento y con el de otros compañeros en la empresa (knowledge sharing).

\section{Empresas multinacionales en la economía local y capacidad de absorción de empresas locales}

Una línea relativamente poco desarrollada y en la cual se centra el interés de nuestro trabajo es si la CA de las empresas locales puede verse afectada positivamente por contratar exempleados de EMN.

Como es sabido, cuando una EMN opera en un determinado país puede convertirse en una fuente de conocimiento para el empresariado local. Este conocimiento se traslada de la EMN hacia las empresas locales de dos formas: por mecanismos de transferencia del conocimiento (acuerdos comerciales, alianzas, intercambio de mejores prácticas, acceso a tecnología, etc.) y como derrames de conocimiento (Spencer, 2008). La diferencia radica en que por los derrames de conocimiento la EMN no recibe ningún tipo de compensación, constituyéndose esto en una 
externalidad positiva para la economía anfitriona (Javorcik, 2004; Görg y Strobl, 2005; Smeets, 2008; Zhang et al., 2010).

Esta relación teórica entre conocimiento derramado desde las EMN hacia las empresas locales ha sido estudiada empíricamente con resultados diversos. Algunos trabajos (Blomström, 1986; Buckley, Clegg y Wang, 2007; Tian, 2007; Wei y Liu, 2006) hallaron resultados positivos (por ejemplo en términos de incremento de la productividad de las empresas del país anfitrión), mientras que otros muestran resultados negativos (Aitken y Harrison, 1999; Feinberg y Majumdar, 2001).

Buscando comprender esta diversidad de resultados, Smeets (2008) señala la importancia de comprender bien y modelar la forma en que operan los canales por medio de los que se pueden producir dichos derrames de conocimiento. De hecho, citando a Görg y Strobl (2005), señala que en muchos casos los investigadores solo se preocupan de encontrar una relación estadística (positiva o negativa) entre el desempeño de las empresas locales y la existencia de EMN en el país anfitrión, lo que supone implícitamente que los derrames de conocimiento suceden dentro de una «caja negra».

En un reciente estudio de Farole y Winkler (2014) se presenta un interesante marco conceptual basado en un trabajo previo de Paus y Gallagher (2008), para explicar la dinámica de los derrames de conocimiento desde las EMN hacia las empresas locales. Este marco identifica los factores que median para la existencia de derrames de conocimiento asociados con la operación de EMN en un país anfitrión.

De acuerdo con esos autores, los elementos determinantes para el surgimiento de derrames de conocimiento se pueden agrupar en tres categorías. En primer lugar, las características de las $E M N$, tales como motivos para el establecimiento de operaciones en el país anfitrión, las estrategias de producción y abastecimiento global, modelos de entrada, así como el tiempo de presencia en el país anfitrión. En segundo lugar, la capacidad de las empresas nacionales para absorber el conocimiento y la tecnología desde las EMN (capacidad de absorción) por medio de los tres canales descritos por Saggi (2002). Finalmente, los factores y el marco institucional del país anfitrión, tales como la regulación del mercado laboral, los derechos de propiedad intelectual, el acceso al financiamiento, y la infraestructura para el aprendizaje y la innovación. Un aspecto importante de este marco conceptual es que el conjunto de factores que median para el derrame de conocimiento de las multinacionales a las empresas locales es dinámico, no estático, toda vez que su composición puede cambiar a través del tiempo.

En síntesis, tanto el trabajo de Farole y Winkler (2014) como otros previos (Paus y Gallagher, 2008; Lim, 2001) señalan la importancia de tomar en cuenta que la relación de aprendizaje entre empresas locales y EMN depende, entre otras cosas, de la CA de las primeras. En otras palabras, que la CA influye en el aprovechamiento que la empresa local pueda hacer del conocimiento que se derrama desde la EMN. Nuestro estudio toma en cuenta este aspecto, pero trata de contestar una pregunta aún poco estudiada: ¿cómo influye en la CA de las empresas locales contratar empleados que hayan trabajado previamente en EMN? La respuesta a esta pregunta constituye un elemento fundamental para comprender la dinámica del conocimiento entre las EMN y el conglomerado empresarial local.

\section{Metodología}

\section{Muestra}

Los datos utilizados provinieron de la encuesta que dio origen al segundo estudio nacional de las mipymes que efectuó el Observatorio Costarricense de las Mipymes (Omipymes) (Gómez, 
2012). En dicho estudio se entrevistó telefónicamente a 1,167 micros, pequeñas y medianas empresas costarricenses ${ }^{1}$ por medio de una muestra probabilística y estratificada por tamaño y sector productivo de la empresa (agricultura-pesca; comercio; servicios, e industria). El error de muestreo máximo en la estimación de proporciones, con un nivel de confianza del 95\%, es de \pm 2.9 puntos porcentuales.

\section{Fuentes de datos secundarios y variables}

En el presente estudio, la variable de interés fue la CA. Para ello usamos el índice de capacidad de absorción (ICA) estimado por Monge-González et al. (2015) para las micros, pequeñas y medianas empresas costarricenses, como la variable dependiente. Dicho ICA fue elaborado a partir del enfoque de Vega-Jurado et al. (2008), proponiéndose un índice compuesto por tres factores: conocimiento organizacional, formalización y mecanismos de integración social. Las otras variables incluidas en el modelo fueron las siguientes. Una es si la mipyme local cuenta con empleados que previamente hubieran trabajado con alguna empresa multinacional radicada en Costa Rica. Otra fue la cantidad de empleados que poseía la mipyme local. Asimismo, se incluyó en el modelaje el sector en el cual operaban las mipymes. El análisis de las variables se efectuó por medio del modelo que se explica a continuación.

\section{Análisis estadísticos de datos}

Para explicar el comportamiento del ICA, se formula una ecuación que trata de medir el efecto de la contratación de exempleados de EMN por parte de empresas locales sobre la CA de estas últimas.

$$
I C A_{i}=\beta_{0}+\beta_{1} \operatorname{ex} L_{i}+\beta_{2} L_{i}+\sum_{j=1}^{3} \beta_{3 j} S_{i j}+\varepsilon_{i}
$$

En el anterior modelo, el subíndice i denota la i-ésima empresa y varía de 1 a 1,167; exL $\mathrm{L}_{\mathrm{i}}$, es una variable que toma el valor de 1 si la empresa i-ésima tiene exempleados de empresas EMN y cero en otro caso; $\mathrm{L}_{\mathrm{i}}$ es el número de empleados que posee la empresa i-ésima y, y $\mathrm{S}_{\mathrm{ij}}$ son tres variables que toman valor 1 si la empresa pertenece al sector j-ésimo y cero en otro caso. El subíndice $\mathrm{j}=1$ denota el sector servicios, $\mathrm{j}=2$ a comercio, $\mathrm{j}=3$ a industria manufacturera y $\mathrm{j}=4$ al sector agricultura y pesca, que al no tomarse en cuenta en el modelo nos indica que es el sector de referencia. La perturbación aleatoria es representada por $\varepsilon_{i}$, que se supone independiente, idénticamente, distribuida normal, con media cero y varianza constante.

En la ecuación (1) el coeficiente de interés es $\beta_{1}$, el cual mide el efecto sobre la CA de la empresa local de contar con exempleados de EMN. En términos generales, mide en cuántos puntos porcentuales aumenta o disminuye el ICA de la empresa local por contar con exempleados de EMN, donde la premisa es que el conocimiento previamente adquirido por estos trabajadores mientras laboraron en una EMN puede ser utilizado en sus nuevas funciones en la empresa local $\mathrm{y}$, por ende, afectar positivamente el desempeño de esta última.

La ecuación (1) se estima por medio de mínimos cuadrados ordinarios (MCO). Cabe señalar que no se incluyeron más variables control, toda vez que para la construcción del IC se tomaron

\footnotetext{
${ }^{1}$ Empresas que contratan menos de 100 empleados por año.
} 
en cuenta muchas características de las empresas locales que si se tomaran en cuenta en el modelo lo invalidarían, pues estarían de ambos lados de la ecuación.

Varias pruebas estadísticas fueron llevadas a cabo para verificar que los supuestos del modelo de regresión con MCO se cumplieran.

Para cada uno de los modelos estimados se verificaron en forma gráfica los supuestos. Para verificar la normalidad de los supuestos se obtuvieron los histogramas de los residuales, así como el gráfico de probabilidad normal. Y para verificar la independencia de los errores, la varianza constante y la media cero se utilizó el gráfico de residuales contra predichos. Esto nos lleva a concluir que las estimaciones de los parámetros de los modelos, así como sus errores estándar, poseen todas las características que brindan los estimadores mínimos cuadráticos.

\section{Resultados}

El primer punto por responder es qué tan importante es la cantidad de exempleados de multinacionales contratados por empresas locales en Costa Rica. La tabla 1 muestra los resultados a esta pregunta para toda la muestra y por sector productivo. Como puede observarse en la columna seis, el $16.28 \%$ de todas las empresas locales señalaron contar con exempleados de EMN en el año 2011. Este porcentaje es mucho mayor en el caso del sector manufactura (18.39\%) y mucho menor en el de agricultura y pesca $(10.82 \%)$.

En cuanto a la composición de la muestra según tamaño de las empresas y su distribución por sector productivo, las cifras de la tabla 2 permiten afirmar que la mitad de las empresas tienen un tamaño igual o inferior a 10 empleados, que el tamaño de las empresas va desde un empleado hasta 540, que en promedio tienen cerca de 27 empleados y con una desviación estándar cercana a 42 empleados. Los sectores con empresas más grandes son servicios y comercio (540 y 400 empleados, respectivamente).

Tabla 1

Costa Rica. Índice de capacidad de absorción según sector productivo

\begin{tabular}{lclllll}
\hline Sector productivo & Observaciones & Mínimo & Máximo & Mediana & Promedio & Desviación estándar \\
\hline Servicios & 432 & 0.00 & 1.00 & 0.4167 & 0.4284 & 0.21763 \\
Comercio & 318 & 0.00 & 1.00 & 0.4048 & 0.4192 & 0.21109 \\
Manufactura & 223 & 0.00 & 1.00 & 0.3929 & 0.4158 & 0.19146 \\
Agricultura y pesca & 194 & 0.00 & 1.00 & 0.3036 & 0.3276 & 0.20939 \\
Total & 1,167 & 0.00 & 1.00 & 0.3929 & 0.4067 & 0.21244 \\
\hline
\end{tabular}

Fuente: elaboración propia con base en datos de Monge-González et al., 2015.

Tabla 2

Costa Rica. Empresas locales que contratan exempleados de multinacionales

\begin{tabular}{lclllll}
\hline Sector productivo & Observaciones & Mínimo & Máximo & Mediana & Promedio & Desviación estándar \\
\hline Servicios & 432 & 0.00 & 1.00 & 0.0000 & 0.1690 & 0.37517 \\
Comercio & 318 & 0.00 & 1.00 & 0.0000 & 0.1730 & 0.37881 \\
Manufactura & 223 & 0.00 & 1.00 & 0.0000 & 0.1839 & 0.38824 \\
Agricultura y pesca & 194 & 0.00 & 1.00 & 0.0000 & 0.1082 & 0.31150 \\
Total & 1,167 & 0.00 & 1.00 & 0.0000 & 0.1628 & 0.36935 \\
\hline
\end{tabular}

Fuente: elaboración propia basado en la encuesta del Observatorio de Mipymes (Gómez, 2012). 
Tabla 3

Costa Rica. Distribución de las empresas locales según tamaño y sector productivo

\begin{tabular}{lcccccc}
\hline Sector productivo & Observaciones & Mínimo & Máximo & Mediana & Promedio & Desviación estándar \\
\hline Servicios & 425 & 1.00 & 540.00 & 10.00 & 29.76 & 51.75 \\
Comercio & 317 & 1.00 & 400.00 & 10.00 & 25.31 & 38.81 \\
Manufactura & 222 & 2.00 & 150.00 & 10.50 & 25.15 & 30.55 \\
Agricultura y pesca & 194 & 1.00 & 240.00 & 10.00 & 23.95 & 35.86 \\
Total & 1,158 & 1.00 & 540.00 & 10.00 & 26.68 & 42.32 \\
\hline
\end{tabular}

Fuente: elaboración propia basado en la encuesta del Observatorio de Mipymes (Gómez, 2012).

Es importante recalcar que la distribución muestral por sector es similar a la poblacional en 2011, y que para llevar a cabo el análisis de regresión lineal por sector se cuenta con tamaños de muestra suficientemente grandes.

Como se indicó con anterioridad, la variable dependiente en la ecuación (1) es el ICA construido por Monge-González et al. (2015), para un grupo de 1,167 micros, pequeñas y medianas empresas costarricenses, el cual agrupa una serie de variables en las categorías propuestas por Vega-Jurado et al. (2008). La tabla 3 muestra algunas medidas estadísticas resumen del ICA para toda la muestra, así como por sector productivo.

Tal y como se observa en la tabla 3, el promedio del ICA es de 0.4067 y una desviación estándar de 0.2124. Se presenta una baja importante en el promedio del sector agricultura y pesca (0.3276). Los otros tres sectores productivos muestran valores promedio muy similares. En todos los sectores hay empresas con valores muy altos (uno) y muy bajos (cero) para el ICA. En otras palabras, existe una importante variabilidad en los valores del ICA en toda la muestra.

Los resultados de la estimación de la ecuación (1) para toda la muestra se presentan en la tabla 4. En la segunda columna de dicha tabla se observa que el coeficiente asociado con la variable ICA es de 0.09 positivo y significativamente diferente de cero $(p=0.0000)$. Esto significa que la contratación de exempleados de EMN por parte de las empresas locales incrementa en nueve puntos porcentuales el ICA de conocimiento de dichas empresas.

Con base en el anterior resultado y dado que una de las políticas de las autoridades gubernamentales costarricenses ha sido la atracción de inversión extranjera, se podría afirmar que dicha política tiene una externalidad positiva en términos de derrames de conocimiento hacia las empresas locales, al incrementar su CA de conocimiento y tecnología.

Por otra parte, se observa además que los coeficientes asociados con todas las otras variables en la ecuación (número de empleados y las variables dummy de los sectores productivos) son positivos y significativos estadísticamente, lo cual señala la importancia de dividir el análisis por sector.

Al estimar la ecuación (1) para cada uno de los cuatro sectores productivos, se encuentran resultados interesantes, los cuales se muestran en las columnas 3 a 6 de la tabla 4 . De allí se puede concluir que la contratación de exempleados de EMN tiene un efecto positivo sobre el ICA de conocimiento en las empresas de todos los sectores productivos, pero con mayor impacto en las del sector servicios (0.109), seguido de las empresas del sector agricultura y pesca (0.090) y del sector comercio (0.085), y que en el sector manufactura es donde se tiene un efecto menor (0.059). En todos los casos los coeficientes son positivos y estadísticamente diferentes de cero, aunque en el caso de los sectores de comercio y servicios el grado de significación es mayor.

Estos resultados contribuyen a la teoría desde diversas perspectivas. Por un lado aportan en cuanto a los factores que influyen para que las empresas incrementen su CA. Existe evidencia que 
Tabla 4

Costa Rica: impacto de los exempleados de multinacionales en la capacidad de absorción de las empresas locales

\begin{tabular}{|c|c|c|c|c|c|}
\hline \multirow[t]{2}{*}{ Variables } & \multirow[t]{2}{*}{ Todos los sectores } & \multicolumn{4}{|c|}{ Sectores } \\
\hline & & $\begin{array}{l}\text { Agricultura } \\
\text { y Pesca }\end{array}$ & Comercio & Manufactura & Servicios \\
\hline $\begin{array}{l}\text { Empresa tiene empleados que } \\
\text { vienen de multinacionales }\end{array}$ & $0.090^{* * *}(0.000)$ & $\begin{array}{l}0.090^{*} \\
(0.066)\end{array}$ & $\begin{array}{l}0.085^{\text {**** }} \\
(0.006)\end{array}$ & $\begin{array}{l}0.059^{*} \\
(0.079)\end{array}$ & $\begin{array}{l}0.109^{* * *} \\
(0.000)\end{array}$ \\
\hline $\begin{array}{l}\text { ¿Cuántas personas se } \\
\text { encuentran trabajando } \\
\text { actualmente en su/la } \\
\text { empresa? }\end{array}$ & $0.000^{* * *}(0.001)$ & $\begin{array}{l}0.000^{*} \\
(0.728)\end{array}$ & $\begin{array}{l}0.001^{*} \\
(0.054)\end{array}$ & $\begin{array}{l}0.001^{* *} \\
(0.021)\end{array}$ & $\begin{array}{l}0.000^{* * *} \\
(0.046)\end{array}$ \\
\hline Empresa pertenece a servicios & $0.093^{* * *}(0.000)$ & & & & \\
\hline $\begin{array}{l}\text { Empresa pertenece a } \\
\text { comercio }\end{array}$ & $0.086^{* * *}(0.000)$ & & & & \\
\hline Empresa pertenece a industria & $0.080^{* * *}(0.000)$ & & & & \\
\hline Constante & $0.307^{* * *}(0.000)$ & $\begin{array}{l}0.314^{* * *} \\
(0.000)\end{array}$ & $\begin{array}{l}0.391^{* * *} \\
(0.000)\end{array}$ & $\begin{array}{l}0.380^{* * * *} \\
(0.000)\end{array}$ & $\begin{array}{l}0.399^{* * *} \\
(0.000)\end{array}$ \\
\hline Observaciones & 1157 & 193 & 316 & 221 & 424 \\
\hline $\mathrm{R}^{2}$ & 0.065 & 0.019 & 0.035 & 0.046 & 0.050 \\
\hline $\mathrm{F}$ & $16.103(0.000)$ & $\begin{array}{l}1.876 \\
(0.156)\end{array}$ & $\begin{array}{l}5.771 \\
(0.003)\end{array}$ & $\begin{array}{l}5.268 \\
(0.006)\end{array}$ & $\begin{array}{l}11.115 \\
(0.000)\end{array}$ \\
\hline
\end{tabular}

La ausencia de asteriscos significa que el coeficiente no es diferente de cero con significación estadística.

Fuente: elaboración propia.

* : coeficiente estadísticamente significativos al $10 \%$.

** : coeficiente estadísticamente significativos al $5 \%$.

${ }^{* * *}:$ coeficiente estadísticamente significativos al $1 \%$.

aspectos como el conocimiento organizacional, la formalización, los mecanismos de integración social, el enfoque estratégico, las capacidades organizacionales y el conocimiento compartido influyen en la CA (Vega-Jurado et al., 2008; Flor et al., 2011; Jansen et al., 2005; Liao et al., 2007). Nuestro trabajo aporta un elemento concreto adicional a los factores que influyen en la CA, como sería la contratación de exempleados de EMN. Asimismo, las diferencias encontradas sectorialmente dejan ver una posible veta de investigación muy interesante, pues ligaría el entorno como un factor a su vez influyente en la CA de las pymes locales, muy de la mano con los resultados de autores como Farole y Winkler (2014).

Por otro lado, nuestro trabajo aborda una línea temática poco estudiada como serían las posibles externalidades positivas de las EMN hacia las mipymes locales por vía de derrames de conocimiento. En ese sentido se uniría a trabajos como los de Blomström, 1986; Buckley et al., 2007; Tian, 2007; Wei y Liu, 2006, quienes aportaron evidencia de externalidades positivas emanadas de las EMN hacia el empresariado del país anfitrión.

\section{Conclusiones}

La evidencia empírica disponible señala que la CA influye sobre el desempeño de las empresas (Wales et al., 2013). Por ende, conocer los factores que influyen a su vez sobre la CA de las empresas es valioso. Por otro lado, en la actualidad muchas naciones han aplicado una estrategia de atracción de inversión extrajera, vía instalación de EMN, en la cual, además de ciertos beneficios tangibles (i.e. generación de empleo), se espera que exista transferencia y derrames de conocimiento de dichas EMN hacia el sector empresarial local. En esta intersección temática 
es en la que se ubicó nuestro estudio. Buscamos responder cómo afecta a la CA de las empresas locales el hecho de contratar empleados provenientes de EMN ubicadas en el mismo país. Lo anterior usando un país como Costa Rica que ha sido reconocido por su estrategia de atracción de inversión extranjera directa (World Economic Forum, 2013).

Los resultados dejan concluir que la contratación de exempleados de EMN por parte de las empresas locales incrementa, en términos generales, en nueve puntos porcentuales el ICA de las empresas locales, con algunas diferencias relevantes según se trate de sectores y tamaños de empresas. Ergo, la contratación de exempleados de EMN por parte de empresas locales constituye un elemento importante para incrementar la CA de conocimiento y tecnología de estas últimas y con ello su productividad y desempeño.

Este resultado implica además una fuerte evidencia de una externalidad positiva de la atracción de inversión extranjera directa que han promovido las autoridades costarricenses durante las últimas décadas.

El trabajo tiene limitaciones como cualquiera de su tipo. En primer lugar debe mencionarse que acudimos a una medición que no contempla cuánto tiempo estuvo y qué tipo de conocimiento adquirió el exempleado en la EMN; solamente tomamos la referencia de si estuvo o no contratado por una EMN. Además, no se analiza la forma en la cual aplicó los conocimientos adquiridos en la EMN al trasladarse a trabajar a la empresa local.

A partir del presente artículo se abren futuras líneas de investigación. Por ejemplo, se requiere más investigación para entender mejor el proceso de aprendizaje de las empresas locales por medio de la contratación de exempleados de EMN, a fin de poder promover políticas que favorezcan la capacitación de estos exempleados en ciertas áreas mientras laboran en las EMN. Asimismo, sería valioso profundizar en las dinámicas del proceso en las mipymes buscando comprender mejor qué factores influyen más en que dichas empresas puedan aprovechar el conocimiento que trae consigo el exempleado de una EMN que se desplaza a trabajar con una empresa local.

\section{Referencias}

Aitken, B. J. y Harrison, A. E. (1999). Do domestic firms benefit from direct foreign investment? Evidence from Venezuela. American Economic Review, 89(3), 605-618.

Blomström, M. (1986). Foreign investment and productive efficiency: The case of Mexico. Journal of Industrial Economics, 35(1), 97-110.

Buckley, P. J., Clegg, J. y Wang, C. (2007). Is the relationship between inward FDI and spillover effects linear? An empirical examination of the case of China. Journal of International Business Studies, 38(3), 447-459.

Camisón, C. y Forés, B. (2010). Knowledge absorptive capacity: New insights for its conceptualization and measurement. Journal of Business Research, 63, 707-715. http://dx.doi.org/10.1016/j.jbusres.2009.04.022

Chen, Y. S., Lin, M. J. J. y Chang, C. H. (2009). The positive effects of relationship learning and absorptive capacity on innovation performance and competitive advantage in industrial markets. Industrial Marketing Management, 38(2), 152-158. http://dx.doi.org/10.1016/j.indmarman.2008.12.003

Cohen, W. M. y Levinthal, D. A. (1990). Absorptive capacity: A new perspective on learning and innovation. Administrative Science Quarterly, 35(1), 128-152.

Cordero, J. y Paus E. (2008). Foreign investment and economic development in Costa Rica: The unrealized potential. Discussion paper number 13, Working Group on Development and Environment in the Americas.

De Jong, J. P. J. y Freel, M. (2010). Absorptive capacity and the reach of collaboration in high technology small firms. Research Policy, 39, 47-54. http://dx.doi.org/10.1016/j.respol.2009.10.003

Escribano, A., Fosfuri, A. y Tribó, J. A. (2009). Managing external knowledge flows: The moderating role of absorptive capacity. Research Policy, 38, 96-105. http://dx.doi.org/10.1016/j.respol.2008.10.022

Farole, T. y Winkler, D. (2014). Making Foreign Direct Investment Work for Sub-Saharan Africa: Local Spillovers and Competitiveness in Global Value Chains. Washington DC: The World Bank. 
Feinberg, S. y Majumdar, S. (2001). Technology spillovers from FDI in the Indian pharmaceutical industry. Journal of International Business Studies, 32(3), 421-438.

Flor, M. L., Oltra, M. J. y García, C. (2011). La relación entre la capacidad de absorción del conocimiento externo y la estrategia empresarial: un análisis exploratorio. Revista Europea de Dirección y Economía de la Empresa, 20(1), 69-88.

George, G., Zahra, S. A., Wheatley, K. K. y Khan, R. (2001). The effects of alliance portfolio characteristics and absorptive capacity on performance. A study of bio-technology firms. The Journal of High Technology Management Research, 12(2), 205-226.

Girma, S. y Görg, H. (2002). Foreign direct investment, spillovers and absorptive capacity: Evidence from quantile regressions. Research paper/Leverhulme Centre for Research on Globalisation and Economic Policy, No. $2002,14$.

Gómez, A. (2012). Informe técnico: Segundo diagnóstico nacional de MIPYMES. Documento de trabajo. Observatorio Costarricense de las Mipymes.

Görg, H. y Strobl, E. (2005). Spillovers from foreign firms through worker mobility: An empirical investigation. Scandinavian Journal of Economics, 107(4), 693-709.

Grant, R. M. (1996). Toward a knowledge-based theory of the firm. Strategic Management Journal, 17, 109-122.

Grimpe, C. y Sofka, W. (2009). Search patterns and absorptive capacity: Low- and high- technology sectors in European countries. Research Policy, 38, 495-506. http://dx.doi.org/10.1016/j.respol.2008.10.006

Jansen, J. P., van den Bosch, F. A. y Volberda, H. W. (2005). Managing potential and realized absorptive capacity: How do organizational antecedents matter? Academy of Management Journal, 48(6), 999-1015. http://dx.doi.org/ 10.5465/AMJ.2005.19573106

Javorcik, B. (2004). Does foreign direct investment increase the productivity of domestic firms? In search of spillovers through backward linkages. American Economic Review, 94(3), 605-627.

Jiménez, M. M., García, V. J. y Molina, L. M. (2011). Validation of an instrument to measure absorptive capacity. Technovation, 31, 190-202.

Liao, J., Welsch, H. y Stoica, M. (2003). Organizational absorptive capacity and responsiveness: An empirical investigation of growth-oriented SMEs. Entrepreneurship Theory and Practice, 28(1), 63-85. http://dx.doi.org/ 10.1111/1540-8520.00032

Liao, S. h., Fei, W. C. y Chen, C. C. (2007). Knowledge sharing, absorptive capacity and innovation capability: An empirical study of Taiwan's knowledge-intensive industries. Journal of Information Science, 33(3), 340-359. http://dx.doi.org/10.1177/0165551506070739

Lichtenthaler, U. (2009). Absorptive capacity, environmental turbulence, and the complementarity of organizational learning processes. Academy of Management Journal, 52(4), 822-846. http://dx.doi.org/10.5465/AMJ.2009.43670902

Lim, E.-G. (2001). Determinants of, and the Relation Between, Foreign Direct Investment and Growth: A Summary of the Recent Literature. IMF Working Paper WP/01/175.

Miles, R. E. y Snow, C. H. C. (1978). Organizational Strategy, Structure and Process. New York: McGraw-Hill.

Monge-González, R. y González-Alvarado C. (2007). The Role and Impact of MNCs in Costa Rica on Skills Development and Training: The Case of Intel Microsoft, and Cisco. Working paper. International Labor Organization, Geneva, Switzerland.

Monge-González, R., Leiva, J. C. y Rodríguez-Álvarez, J. A. (2012). Inversión extranjera directa, movilidad laboral y derrames de conocimiento en Costa Rica. Revista Tecnología en Marcha, 25(5), 103-115. http://dx.doi.org/ 10.18845/tm.v25i5.483

Monge-González, R., Rodríguez-Álvarez, J. A. y Leiva, J. C. (2015). Propuesta de un índice para medir la capacidad de absorción de las mipymes costarricenses. TEC Empresarial, 9(1), 7-18. http://dx.doi.org/10.18845/te.v9i1.2204

Murovec, N. y Prodan, I. (2009). Absorptive capacity, its determinants, and influence on innovation output: cross-cultural validation of the structural model. Technovation, 29(9), 859-872. http://dx.doi.org/10.1016/ j.technovation.2009.05.010

Nonaka, I. (1994). A dynamic theory of organizational knowledge creation. Organization Science, 5(1), 14-37. http://dx.doi.org/10.1287/orsc.5.1.14

Paus, E. y Gallagher, K.P. (2008). Missing links: foreign investment and industrial development in Costa Rica and Mexico. Studies in Comparative International Development. Transaction Periodicals Consortium, Rutgers Univ., New Brunswick, N.J.

Qian, H. y Acs, Z. J. (2013). An absorptive capacity theory of knowledge spillover entrepreneurship. Small Business Economics, 40, 185-197. http://dx.doi.org/10.1007/s11187-011-9368-X

Ragab, M. y Arisha, A. (2013). Knowledge management and measurement: A critical review. Journal of Knowledge Management, 17(6), 873-901. 
Rothaermel, F. T. y Alexandre, M. T. (2009). Ambidexterity in technology sourcing: The moderating role of absorptive capacity. Organization Science, 20(4), 759-780. http://dx.doi.org/10.1287/orsc.1080.0404

Saggi, K. (2002). Trade, foreign direct investment and international technology transfer: A survey. The World Bank Research Observer, 17(2), 191-235. http://dx.doi.org/10.1093/wbro/17.2.191

Smeets, R. (2008). Collecting the pieces of the FDI knowledge spillovers puzzle. The World Bank Research Observer, 19(3), 1-32. http://dx.doi.org/10.1093/wbro/lkn003

Spencer, J. W. (2008). The impact of multinational enterprise strategy on indigenous enterprises: Horizontal spillovers and crowding out in developing countries. Academy of Management Review, 33, 341-361. http://dx.doi.org/10. 5465/AMR.2008.31193230

Teece, D. J., Pisano, G. y Shuen, A. (1997). Dynamic capabilities and strategic management. Strategic Management Journal, 18(7), 509-533, http://dx.doi.org/10.1002/(SICI)1097-0266(199708)18:7\%3C509::AIDSMJ882\%3E3.0.CO;2-Z.

Tian, X. (2007). Accounting for sources of FDI technology spillovers: Evidence from China. Journal of International Business Studies, 38, 147-159. http://dx.doi.org/10.1057/palgrave.jibs.8400245

Vega-Jurado, J., Gutiérrez-Gracia, A. y Fernández-de-Lucio, I. (2008). Analyzing the determinants of firm's absorptive capacity: beyond R\&D. R\&D Management, 38(4), 392-405.

Wales, W. J., Parida, V. y Patel, P. C. (2013). Too much of a good thing? Absorptive capacity, firm performance, and the moderating role of entrepreneurial orientation. Strategic Management Journal, 34, 622-633. http://dx.doi.org/ 10.1002/smj.2026

Wei, Y. y Liu, X. (2006). Productivity spillovers from R\&D, exports and FDI in China's manufacturing sector. Journal of International Business Studies, 37(4), 544-557.

World Economic Forum (2013). Foreign Direct Investment as a Key Driver for Trade, Growth and Prosperity: The Case for a Multilateral Agreement on Investment [consultado el 30 Abr 2015]. Disponible en: http://www3.weforum.org/ docs/GAC13/WEF_GAC_GlobalTradeFDI_FDIKeyDriver_Report_ 2013.pdf.

Zahra, S. A. y George, G. (2002). Absorptive capacity: A review, reconceptualization, and extension. Academy of Management Review, 27(2), 185-203. http://dx.doi.org/10.5465/AMR.2002.6587995

Zahra, S. A. y Hayton, J. C. (2008). The effect of international venturing on firm performance: The moderating influence of ACAP. Journal of Business Venturing, 23(2), 195-220.

Zhang, Y., Li, H., Li, H. y Zhou, H. L. (2010). FDI spillovers in emerging markets and absorptive capacity. Strategic Management Journal, 31, 969-989. 